GANIT J. Bangladesh Math. Soc. (ISSN 1606-3694) 34 (2014) 111-126

\title{
CRITICAL ANALYSIS OF MAGNETOHYDRODYNAMIC JEFFERY-HAMEL FLOW USING Cu-WATER NANOFLUID
}

\author{
Md. S. Alam ${ }^{1}$, M.A.H. Khan ${ }^{2}$, M.A. Alim ${ }^{2}$ \\ ${ }^{1}$ Department of Mathematics, Jagannath University, Dhaka-1100, Bangladesh \\ ${ }^{2}$ Department of Mathematics, Bangladesh University of Engineering and Technology \\ Dhaka-1000, Bangladesh \\ *E-mail: sarwardu75@gmail.com, sarwar@math.jnu.ac.bd \\ Received 16.11.2014 \\ Accepted 06.01.2015
}

\begin{abstract}
The effects of nanoparticles and magnetic field on the nonlinear Jeffery-Hamel flow using $\mathrm{Cu}$ water nanofluid are analysed in the present study. The basic dimensionless governing equations are solved using power series, which is then analysed by applying a semi-numerical analytical technique called Hermite- Padé approximation. The velocity profiles are presented in convergentdivergent channels for various values of nanoparticles solid volume fraction, Hartmann number, Reynolds number and channel angle. The dominating singularity behavior of the problem is analysed numerically and graphically for nanofluid. The critical relationships among the parameters are also performed qualitatively with the effect of $\mathrm{Cu}$-nanoparticles.
\end{abstract}

Keywords: Jeffery-Hamel flow, Magnetohydrodynamic, Nanofluid, Dominating singularity, Critical relationship, Hermite- Padé approximation

\section{Nomenclature}

$\begin{array}{llcl}\alpha & \text { channel angle } & \phi & \text { nanoparticles volume fraction } \\ \eta & \text { dimensionless angle } & \mu & \text { dynamic viscosity } \\ \theta & \text { any angle } & v & \text { kinematic viscosity } \\ \rho & \text { density } & & \\ \text { Subscripts } & & \\ \text { nf } & \text { nanofluid } & \text { s } & \text { nano-solid particle } \\ \mathrm{f} & \text { base fluid } & & \end{array}$

\section{Introduction}

The study of flows in converging-diverging channel is very important due to its industrial, aerospace, chemical, civil, environmental, mechanical and bio- mechanical engineering applications. Various applications of this type of mathematical model are to understand the flow of rivers and canals and the blood flow in the human body. Jeffery [1] and Hamel [2] first studied the two-dimensional steady motion of a viscous fluid through convergent-divergent channels which is called classical Jeffery-Hamel flow in fluid dynamics. Jeffery-Hamel flows are interesting models of the phenomenon of separation 
of boundary layers in divergent channels. These flows have discovered similarity solutions of the Navier-Stokes equations depending on two non-dimensional parameters, the flow Reynolds number and channel angular widths. Fraenkel [3] then investigated the laminar flow in symmetrical channels with slightly curved walls. In his analysis the velocity field of the flow was obtained as a power series in small curvature parameter where the leading term is the Jeffery-Hamel solution. Sobey and Drazin [4] studied some instabilities and bifurcations of two-dimensional Jeffery-Hamel flows using analytical, numerical and experimental methods. Moreover, the steady flow of a viscous incompressible fluid in a slightly asymmetrical channel was studied by Makinde [5]. He expanded the solution into a Taylor series with respect to the Reynolds number and performed a bifurcation study.

The theory of magnetohydrodynamic (MHD) is inducing current in a moving conductive fluid in the presence of magnetic field; such induced current results force on ions of the conductive fluid. The theoretical study of MHD channel has been a subject of great interest due to its extensive applications in designing cooling systems with liquid metals, MHD generators, accelerators, pumps, and flow meters [6,7]. Makinde [8] investigated the MHD flows in convergent- divergent channels which was an extension of the classical Jeffery-Hamel flows to MHD. He interpreted that the effect of external magnetic field works as a parameter in solution of the MHD flows in convergent - divergent channels. A survey of information about this problem can be found in the Esmaili [9]. Recently, the three analytical methods such as Homotopy analysis method, Homotopy perturbation method and Differential transformation method (DTM) were used by Joneidi et al. [10] to find the analytical solution of Jeffery-Hamel flow. Moreover, the models on classical semi-analytical methods have experienced a revival, in connection with the scheme of new hybrid numerical-analytical techniques for nonlinear differential equations, such as Hermite-Padé approximation method, which demonstrated itself as a powerful benchmarking tool and a prospective substitute to traditional numerical techniques in various applications in science and engineering. The classical Jeffery-Hamel problem was extended in Axford [11] to include the effects of external magnetic field on conducting fluid. Motsa et al. [12] found the solution of the nonlinear equation for the MHD JefferyHamel problem by using spectral-homotopy analysis method. Moghimi et al. [13] also solved the Jeffery-Hamel flow problem by using the homotopy perturbation method. Alam and Khan [14] studied the critical behavior of the effect of MHD on Jeffery-Hamel flows. The convergence of critical values and the change in bifurcation graph for flow Reynolds number and channel angle by the positive effect of magnetic parameter and the critical relationship among the parameters were discussed in the study.

Recent advances in nanotechnology have allowed authors to study the next generation heat transfer nanofluids, a term first introduced by Choi [15]. Nanoparticles have unique chemical and physical properties [16] and have better thermal conductivity and radiative heat transfer compared to the base fluid only. Nanofluids are engineered dilute colloidal 
dispersions of nano-sized (less than $100 \mathrm{~nm}$ ) particles in a base-fluid [17]. Anomalous heat transfer and thermal conductivity enhancement of these fluids were observed by many researchers in the past and has been discussed by Das et al. in their review [18]. Rahman et al. [19] investigated numerically the steady boundary layer flow and heat transfer characteristics of nanofluids using Buongiorno's model past a permeable exponentially shrinking/stretching surface with second order slip velocity. The effects of magnetic field and nanoparticle on the Jeffery-Hamel flow using Adomian decomposition method were studied by Sheikholeslami et al. [20]. The velocity inside the divergent channel for different values of Hartmann number and channel angle and the effect of nanoparticle volume fraction on velocity field in absence of magnetic field was shown in their analysis. More recently, Moradi et al. [21] investigate the effect of three types of nanoparticles $\mathrm{Cu}, \mathrm{TiO}_{2}$ and $\mathrm{Al}_{2} \mathrm{O}_{3}$ on Jeffery-Hamel flow using Differential Transformation Method (DTM). They found that the influence of solid volume fraction of nanoparticles on the velocity and skin friction was more enunciated when compared with the type of nanoparticles. Also, the skin friction coefficient for $\mathrm{Al}_{2} \mathrm{O}_{3}$ was observed maximum in comparison to the other two nanoparticles.

Taking into account the significance of nanofluid, MHD Jeffery-Hamel flow is solved using power series in terms channel angle. The series is analysed by using Hermite-Padé approximation to show the velocity profiles with the effect of $\phi, \alpha$ and Hartmann number $H$. The critical values and bifurcation diagrams of channel angle and flow Reynolds number with the effect of $\phi$ is studied numerically and graphically. The critical relationships among the parameters are also shown to interpret the effect of $\mathrm{Cu}$ nanoparticles solid volume fraction.

\section{Mathematical formulation}

Consider a steady two-dimensional laminar incompressible viscous $\mathrm{Cu}$-water nanofluid flow from a source or sink between two channel walls intersect at an angle $2 \alpha$. A cylindrical coordinate system $(r, \theta, z)$ is used and assumed that the velocity is purely radial and depends on $r$ and $\theta$ so that there is no change in the flow parameter along the $\mathrm{z}$ direction. Further it is presumed that there is an external magnetic field acting vertically downward to the top wall. Let $\alpha$ be the semi-angle and the domain of the flow be $-|\alpha|<\theta<|\alpha|$. Then the governing equations for the flow can be written as Khan [22]

$$
\begin{aligned}
& \frac{\rho_{n f}}{r} \frac{\partial}{\partial r}(r u)+\frac{\rho_{n f}}{r} \frac{\partial}{\partial \theta}(v)=0, \\
& u \frac{\partial u}{\partial r}+\frac{v}{r} \frac{\partial u}{\partial \theta}-\frac{v^{2}}{r}=-\frac{1}{\rho_{n f}} \frac{\partial p}{\partial r}+v_{n f}\left(\nabla^{2} u-\frac{u}{r^{2}}-\frac{2}{r^{2}} \frac{\partial v}{\partial \theta}\right)-\frac{\sigma B_{0}{ }^{2}}{\rho_{n f} r^{2}} u,
\end{aligned}
$$




$$
u \frac{\partial v}{\partial r}+\frac{v}{r} \frac{\partial v}{\partial \theta}+\frac{u v}{r}=-\frac{1}{\rho_{n f} r} \frac{\partial p}{\partial \theta}+v_{n f}\left(\nabla^{2} v-\frac{v}{r^{2}}+\frac{2}{r^{2}} \frac{\partial u}{\partial \theta}\right)
$$

where $\nabla^{2}=\frac{\partial^{2}}{\partial r^{2}}+\frac{1}{r} \frac{\partial}{\partial r}+\frac{1}{r^{2}} \frac{\partial^{2}}{\partial \theta^{2}}$

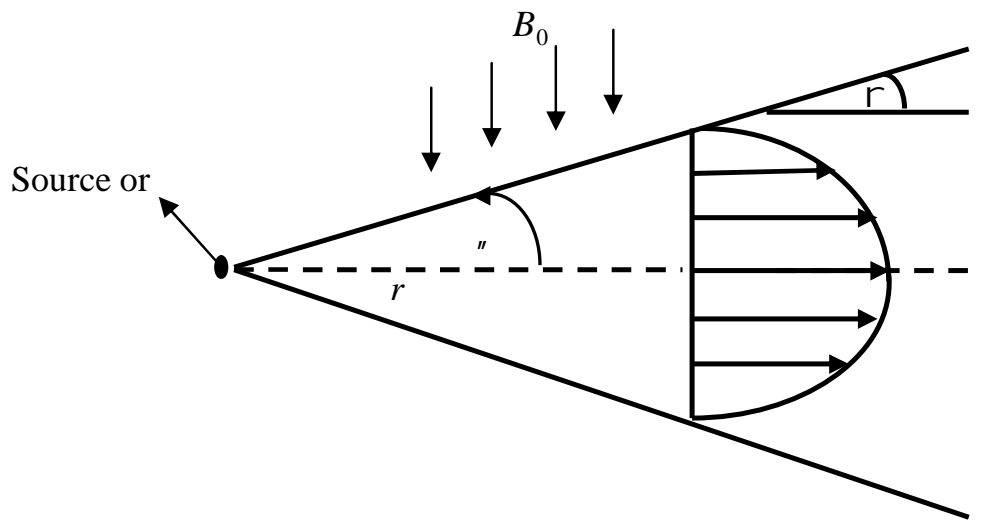

Fig. 1: Geometry of the problem

Assume a symmetric radial flow, so that $v=0$. Also the volumetric flow rate through the channel is

$$
Q=\int_{-\alpha}^{\alpha} u r d \theta
$$

The boundary conditions are

$$
u=0 \text { at } \theta= \pm \alpha
$$

Where $B_{0}$ is the electromagnetic induction, $\sigma$ is the conductivity of the fluid, $u$ is the velocity along radial direction and $p$ is the fluid pressure. The effective density $\rho_{n f}$, the effective dynamic viscosity $\mu_{n f}$, and the kinematic viscosity $v_{n f}$ of the nanofluid are given as Aminossadati and Ghasemi [23].

$$
\rho_{n f}=\rho_{f}(1-\phi)+\rho_{s} \phi, \quad \mu_{n f}=\frac{\mu_{f}}{(1-\phi)^{2.5}}, v_{n f}=\frac{\mu_{n f}}{\rho_{n f}},
$$

Here, $\phi$ is the solid volume fraction of the nanoparticles.

If it requires $Q \geq 0$, then for $\alpha>0$ the flow is diverging from a source at $r=0$.

Let $\psi=\psi(r, \theta)$ be the stream function, then

$$
\partial \psi / \partial \theta=u r, \quad \partial \psi / \partial r=0
$$


Introducing the dimensionless variables

$$
\eta=\theta / \alpha \text { and } F(\eta ; \operatorname{Re}, H, \phi, \alpha)=\psi(\theta) / Q,
$$

The Navier-Stokes equations (2-3) reduce to the ordinary differential equation as Alam and Khan [14]

$$
F^{(i v)}+2 \alpha \operatorname{Re} A(1-\phi)^{2.5} F^{\prime} F^{\prime \prime}+\left(4-(1-\phi)^{1.25} H\right) \alpha^{2} F^{\prime \prime}=0
$$

The boundary conditions are as follows:

$$
\begin{aligned}
& F= \pm 1, \quad F^{\prime}=0 \text { at } \eta= \pm 1 \\
& A=(1-\phi)+\frac{\rho_{s}}{\rho_{f}} \phi, \quad \operatorname{Re}=\frac{Q}{v_{f}}, \quad H=\sqrt{\frac{\sigma B_{0}{ }^{2}}{\rho_{f} v_{f}}},
\end{aligned}
$$

Where $A$ is a parameter, Hartmann number $H$, flow Reynolds number $R e$, channel angle $\alpha$ and nanoparticles solid volume fraction $\phi$.

\section{Series analysis}

The following power series expansion is considered in terms of the parameter $\alpha$ as equation (6) is non-linear for velocity field

$$
F(\eta)=\sum_{i=0}^{\infty} F_{i}(\eta) \alpha^{i}, \text { as }|\alpha|<1
$$

The non-dimensional governing equations are then solved into series solution by substituting the Eq. (9) into Eqs. (6) and (7) and equating the coefficients of powers of $\alpha$.

Order zero $\left(\alpha^{0}\right)$ :

$$
\begin{aligned}
& \frac{d^{4} F_{0}(\eta)}{d \eta^{4}}=0, \\
& F_{0}( \pm 1)= \pm 1, F_{0}^{\prime}( \pm 1)=0,
\end{aligned}
$$

Order one $\left(\alpha^{1}\right)$ :

$$
\begin{aligned}
& \frac{d^{4} F_{1}(\eta)}{d \eta^{4}}+2 \operatorname{Re} A(1-\phi)^{2.5} \frac{d F_{0}(\eta)}{d \eta} \frac{d^{2} F_{0}(\eta)}{d \eta^{2}}=0, \\
& F_{1}( \pm 1)=0, F_{1}^{\prime}( \pm 1)=0,
\end{aligned}
$$

With the help of algebraic programming language MAPLE, we have computed the first 18 coefficients for the series of the velocity $F(\eta)$ in terms of $\alpha, H, R e, \phi, A$. The first few coefficients of the series for $F(\eta)$ are as follows: 


$$
\begin{aligned}
& F(\eta ; \alpha, \operatorname{Re}, H, \phi, A)=\frac{3}{2} \eta-\frac{1}{2} \eta^{3}-\frac{3}{280} \operatorname{Re} A(1-\phi)^{(5 / 2)} \eta\left(\eta^{2}-5\right)(\eta-1)^{2}(\eta+1)^{2} \alpha \\
& +\frac{1}{431200} \eta(\eta-1)^{2}(\eta+1)^{2}\left(43120+9590 \eta^{4} \operatorname{Re}^{2} A^{2} \phi^{2}-9590 \eta^{4} \operatorname{Re}^{2} A^{2} \phi^{3}\right. \\
& +4795 \eta^{4} \operatorname{Re}^{2} A^{2} \phi^{4}-959 \eta^{4} \operatorname{Re}^{2} A^{2} \phi^{5}-4795 \eta^{4} \operatorname{Re}^{2} A^{2} \phi-24720 \eta^{2} \operatorname{Re}^{2} A^{2} \phi^{2} \\
& +24720 \eta^{2} \operatorname{Re}^{2} A^{2} \phi^{3}-12360 \eta^{2} \operatorname{Re}^{2} A^{2} \phi^{4}+2472 \eta^{2} \operatorname{Re}^{2} A^{2} \phi^{5}+12360 \eta^{2} \operatorname{Re}^{2} A^{2} \phi \\
& -980 \eta^{6} \operatorname{Re}^{2} A^{2} \phi^{2}+80 \eta^{6} \operatorname{Re}^{2} A^{2} \phi^{3}-490 \eta^{6} \operatorname{Re}^{2} A^{2} \phi^{4}+98 \eta^{6} \operatorname{Re}^{2} A^{2} \phi^{5}+490 \eta^{6} \operatorname{Re}^{2} A^{2} \phi \\
& +2875 \operatorname{Re}^{2} A^{2}-10780 H(1-\phi)^{(1 / 4)}+28750 \operatorname{Re}^{2} A^{2} \phi^{2}-28750 \operatorname{Re}^{2} A^{2} \phi^{3}-98 \eta^{6} \operatorname{Re}^{2} A^{2} \\
& -2472 \eta^{2} \operatorname{Re}^{2} A^{2}+959 \eta^{4} \operatorname{Re}^{2} A^{2}+10780 H(1-\phi)^{(1 / 4)} \phi-2875 \operatorname{Re}^{2} A^{2} \phi^{5}-14375 \operatorname{Re}^{2} A^{2} \phi \\
& \left.+14375 \operatorname{Re}^{2} A^{2} \phi^{4}\right) \alpha^{2}+O\left(\alpha^{3}\right)
\end{aligned}
$$

Applying differential and algebraic approximant methods to the series we determine the comparison between the present and previous solutions and the changes in bifurcation graphs for the channel angle and flow Reynolds number by the positive effect of nanoparticle volume fraction. The effect of magnetic field and nanofluid on velocity field are also shown graphically using differential approximant method. The details of Hermite-Pade' approximants method are described below.

\section{Computational procedure: Hermite-Padé approximants.}

The idea of channel angle criticality or non-existence of steady-state solution to nonlinear boundary layer equations for certain parameter values are extremely important from physical point of view. To compute the critical behaviour of the system, we shall employ here a very efficient solution method, known as Hermite-Padé approximants, which was first introduced by Padé [24] and Hermite [25].

Assume that the partial sum for the function $S(\alpha)$ is

$$
S_{N}(\alpha)=\sum_{n=0}^{N-1} a_{n} \alpha^{n}, \quad|\alpha|<1
$$

Because of the continuation of analytical solution and dominating singularity behavior, the bifurcation study is performed using the partial sum (15). The dominating behavior of the function $S(\alpha)$ represented by a series (15) may be written as

$$
S(\alpha) \sim \begin{cases}B+A\left(1-\frac{\alpha}{\alpha_{c}}\right)^{\delta} & \text { when } \delta \neq 0,1,2, \ldots, \\ B+A\left(1-\frac{\alpha}{\alpha_{c}}\right)^{\delta} \ln \left|1-\frac{\alpha}{\alpha_{c}}\right| & \text { when } \delta=0,1,2, \ldots,\end{cases}
$$

as $\alpha \rightarrow \alpha_{c}$, where $A$ and $B$ are some constants and $\alpha_{c}$ is the critical point with the critical exponent $\delta$. 
Assume that the $(d+1)$ tuple of polynomials, where $d$ is a positive integer:

$$
P_{N}^{[0]}, P_{N}^{[1]}, \ldots, P_{N}^{[d]}
$$

where, $\quad \operatorname{deg} P_{N}^{[0]}+\operatorname{deg} P_{N}^{[1]}+\ldots+\operatorname{deg} P_{N}^{[d]}+d=N$,

is a Hermite-Padé form of these series if

$$
\sum_{i=0}^{d} P_{N}^{[i]}(\alpha) S_{i}(\alpha)=O\left(\alpha^{N}\right) \text { as }|\alpha|<1
$$

Here $S_{0}(\alpha), S_{1}(\alpha), \ldots, S_{d}(\alpha)$ may be independent series or different form of a unique series. We need to find the polynomials $P_{N}^{[i]}$ that satisfy the equations (17) and (18). These polynomials are completely determined by their coefficients. So, the total number of unknowns in equation (18) is

$$
\sum_{i=0}^{d} \operatorname{deg} P_{N}^{[i]}+d+1=N+1
$$

Expanding the left hand side of equation (18) in powers of $\alpha$ and equating the first $N$ equations of the system equal to zero, we get a system of linear homogeneous equations. To calculate the coefficients of the Hermite-Padé polynomials it requires some sort of normalization, such as

$$
P_{N}^{[i]}(0)=1 \text { for some integer } 0 \leq i \leq d
$$

It is important to emphasize that the only input required for the calculation of the Hermite-Padé polynomials are the first $N$ coefficients of the series $S_{0}(\alpha), S_{1}(\alpha), \ldots, S_{d}(\alpha)$. The equation (19) simply ensures that the coefficient matrix associated with the system is square. One way to construct the Hermite-Padé polynomials is to solve the system of linear equations by any standard method such as Gaussian elimination or Gauss-Jordan elimination. In practice, one usually finds that the dominant singularities as well as the possibility of multiple solution branches for the nonlinear problem are located at zeroes of the leading polynomial $P_{N}^{[d]}(\alpha)$ coefficients of the equation (18). If the singularity is of algebraic type, then the exponent $\delta$ may be approximated by

$$
\delta_{N}=d-2-\frac{P_{N}^{[d-1]}\left(\alpha_{c, N}\right)}{D P_{N}^{[d]}\left(\alpha_{c, N}\right)} .
$$

Drazin -Tourigney Approximants [26] is a particular kind of algebraic approximants and Khan [27] introduced High-order differential approximant (HODA) as a special type of differential approximants. High-order partial differential approximants (HPDA) discussed in Rahman [28] is a partial differential approximants. More information about the above mentioned approximants can be found in the respective references. 


\section{Results and Discussions}

The main objective of the current work is to analyze the effect of nanofluid and magnetic field on Jeffery-Hamel flow of viscous incompressible fluid by using Hermite-Padé approximants. The understanding of the flow physics is achieved through a combination of numerical studies. Although there are four parameters of interest in the present problem the effects of nanoparticle volume fraction $\phi$, channel angle $\alpha$, Reynolds number $\mathrm{Re}$ and Hartman number $\mathrm{H}$. The densities of the base fluid and $\mathrm{Cu}$-nanoparticles are respectively $998.1\left(\mathrm{~kg} / \mathrm{m}^{3}\right), 8933\left(\mathrm{~kg} / \mathrm{m}^{3}\right)$.

Table1: Numerical values of critical angles $\alpha_{c}$ and corresponding exponent $\delta_{c}$ at $\operatorname{Re}=20$ and $H=1$ for various values of $\phi$.

\begin{tabular}{cccc}
\hline \multicolumn{2}{c|}{ Present study } & & Fraenkel [3] \\
\hline$\phi$ & $\alpha_{c}$ & $\delta_{c}$ & $\alpha_{c}$ \\
\hline 0 & 0.2691819115000 & 0.49515872313 & 0.269 \\
0.05 & 0.2122678984825 & 0.49785814583 & - \\
0.1 & 0.1963360739593 & 0.50387522948 & - \\
0.2 & 0.1828175409234 & 0.49803815751 & - \\
\hline
\end{tabular}

Table2: Numerical values of critical $\operatorname{Re}_{c}$ and corresponding exponent $\delta_{c}$ at $\alpha=0.1$ and $H=1$ for various values of $\phi$.

\begin{tabular}{crrr}
\hline \multicolumn{2}{c}{ Present study } & & Fraenkel [3] \\
\hline$\phi$ & $\operatorname{Re}_{c}$ & $\delta_{c}$ & $\operatorname{Re}_{c}$ \\
\hline 0 & 54.47285679258 & 0.5071160433 & 54.61 \\
0.05 & 44.31499529952 & 0.4985570114 & - \\
0.1 & 39.49239826450 & 0.4996886803 & - \\
0.2 & 36.73956809792 & 0.4990504907 & - \\
\hline
\end{tabular}

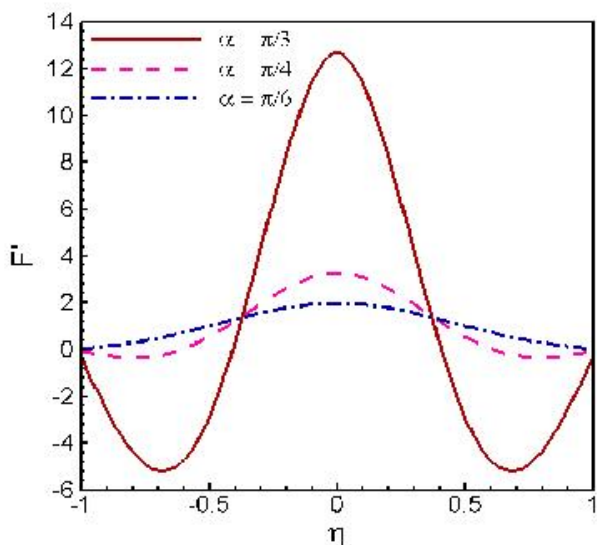

(a) $\phi=0$

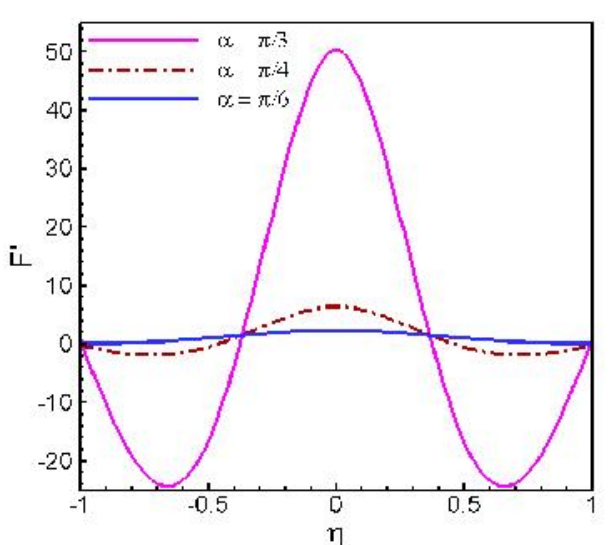

(b) $\phi=0.05$

Fig. 2: Velocity profiles in divergent channel with different values of $\alpha$ at $\operatorname{Re}=7, H=1$ for base fluid and $\mathrm{Cu}$-water nanofluid. 
The series (14) is analyzed by High-order differential approximant method [27] to show the comparison between present results and the results of Fraenkel [3] in Tables 1-2 and the variation in the critical values $\alpha_{c}$ and $\operatorname{Re}_{c}$ with critical exponent $\delta_{c}$ for various values of nanoparticles solid volume fraction significantly. The results of the numerical computations of velocity profiles for different values of the aforementioned parameters are displayed graphically in Figures. (2)-(5). Table 1 exhibits the decreases of critical channel semi-angle $\alpha_{c}$ for four different increasing values of $\phi=0,0.05,0.1,0.2 \mathrm{as} \mathrm{Cu}$ nanoparticles, where water is the base fluid by considering $d=4$ and $N=18$. The values of $\delta_{c}$ confirm that $\alpha_{c}$ is a branch point using HODA. Moreover, Table 2 implies that $\operatorname{Re}_{c}$ decreases both significantly and uniformly for different increasing values of $\phi$ and $\operatorname{Re}_{c}$ is a branch point verified by the values of $\delta_{c}$. The results of Tables 1-2 show a good agreement with those results of Fraenkel [3] for $\phi=0$.

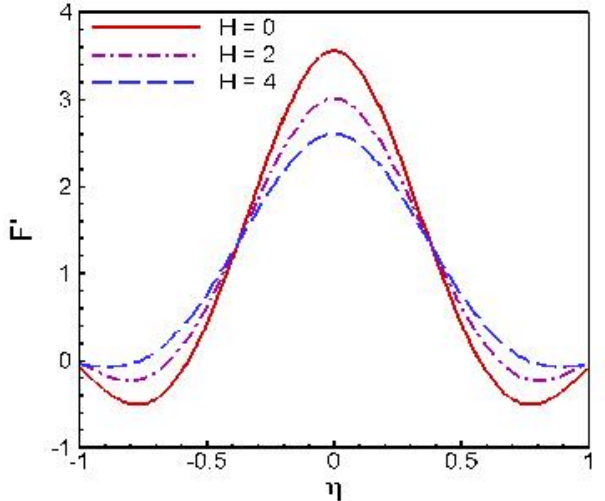

(a) $\phi=0$

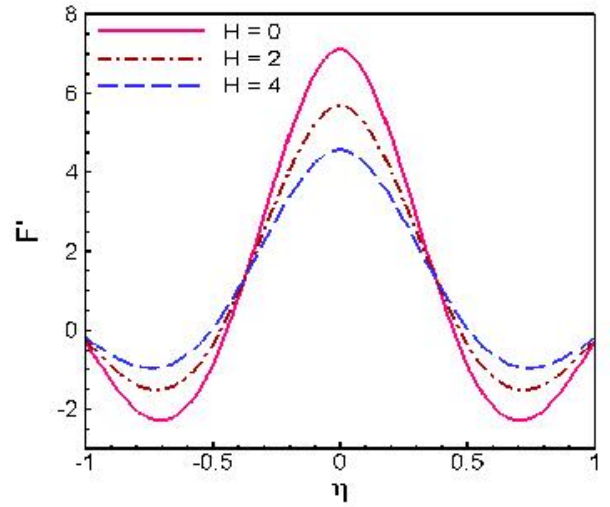

(b) $\phi=0.05$

Fig. 3: Velocity profiles in divergent channel with different values of $\mathrm{H}$ at $\mathrm{Re}=7, \alpha=\pi / 4$ for base fluid and $\mathrm{Cu}$-water nanofluid.

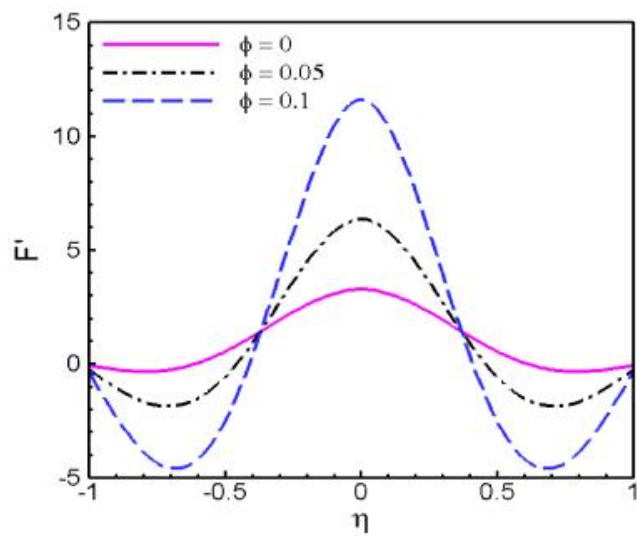

(a) Divergent channel $(\alpha=\pi / 4)$

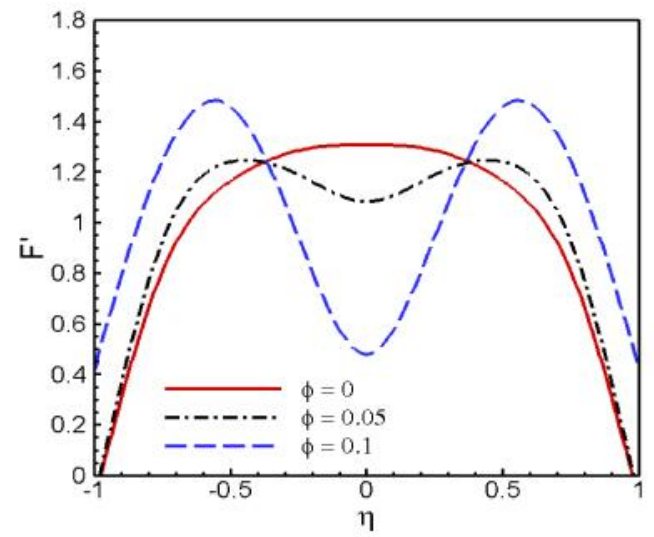

(b) Convergent channel $(\alpha=-\pi / 4)$

Fig. 4: Velocity profiles with different values of $\phi$ at $\mathrm{Re}=7, \mathrm{H}=1$ for $\mathrm{Cu}$-water nanofluid. 
Figures 2 and 3 show the effects of channel angle and magnetic field on the velocity profiles in divergent channel for both viscous base fluid and nanofluid respectively. It is seen from Figure 2(a) that the velocity increases moderately with rising values of $\alpha$ at $\operatorname{Re}=7, H=1$ for viscous fluid $(\phi=0)$ but the differences between velocity profiles are more noticeable at larger angles. However, the backflow is detected in diverging channel for higher values of $\alpha=\pi / 3$. In Figure 2(b) the effect of $(\phi=0.05)$ accelerates the increment of centerline velocities more rapidly, while there occurs major backflow near the walls at large value of $\alpha=\pi / 3$. The flow breaks the symmetry, with most of the fluid going in a thin layer along the walls. The fluid is prevented from utilizing the whole area of the expanding channel by a recirculation vortex which blocks the exit. In addition, secondary instabilities driven by this vertical motion develop in this flow.

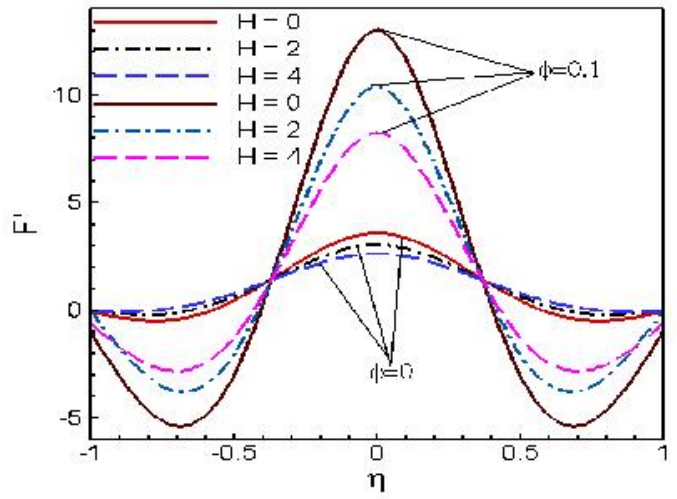

Fig. 5: Combined effects of Hartmann number and solid volume fraction of $\mathrm{Cu}$-nanoparticles on velocity profile for $\alpha=\pi / 4, \operatorname{Re}=7$.

The velocity curves in Figure 3(a) show that the rate of alteration is significantly and uniformly reduced with increase of Hartmann number $H$ in absence of nanoparticles $(\phi=0)$. The transverse magnetic field opposes the alteration phenomena clearly. The variation of $H$ leads to the variation of the Lorentz force due to magnetic field and the Lorentz force produces more resistance to the alternation phenomena.

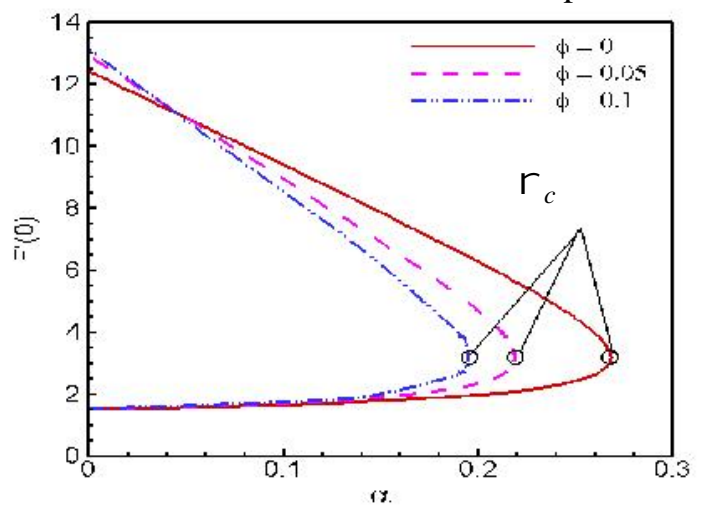

Fig. 6: Approximate bifurcation diagram of $\alpha$ in the $\left(\alpha, \mathrm{F}^{\prime}(0)\right)$ plane at $\mathrm{H}=1, \mathrm{Re}=20$ with different $\phi$ obtained by Drazin-Tourigny method [26] for $d=4$. 


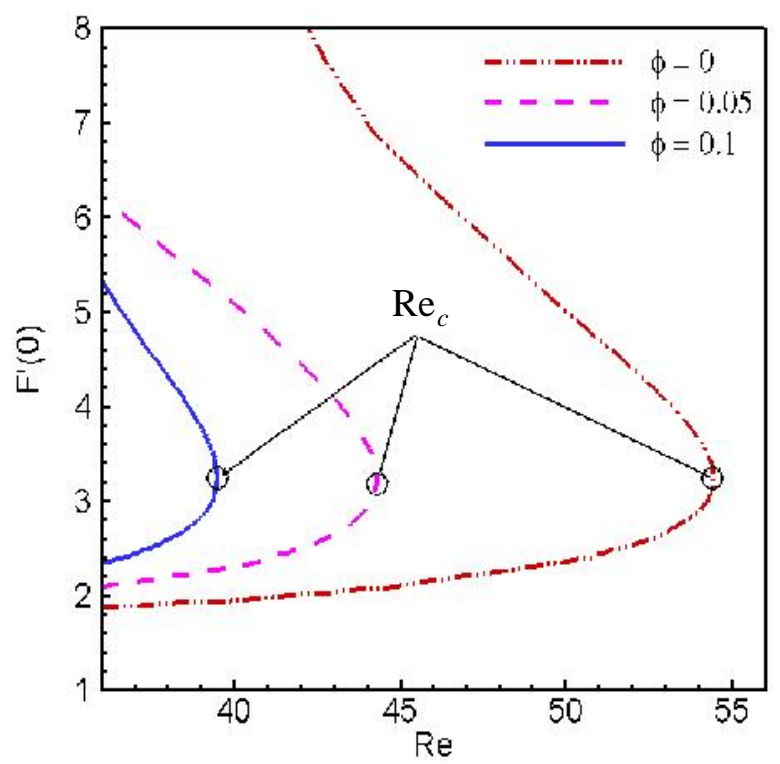

Fig. 7: Approximate bifurcation diagram of $\mathrm{Re}$ in the $\left(\mathrm{Re}, \mathrm{F}^{\prime}(0)\right)$ plane at $\mathrm{H}=1, \alpha=0.1$ with different $\phi$ obtained by Drazin-Tourigny method [26] for $\mathrm{d}=4$.

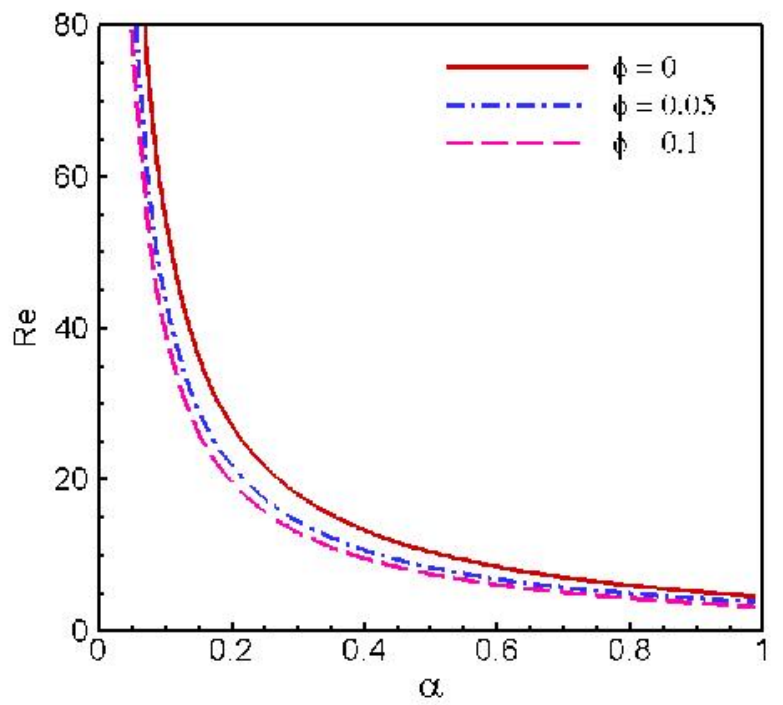

Fig. 8: Critical relation between $\alpha$ and $R e$ for different values of $\phi$ at $\mathrm{H}=1$ obtained by HPDA [28] for $\mathrm{d}=5$.

It can be noted from Figure 3(b) that the centerline velocity increases reasonably for nanofluid $(\phi=0.05)$ than viscous fluid with dropping $H$ at a small angle $\alpha=0.1$. Figure 4 represents the consequences of solid volume fraction on velocity profile at $\alpha=0.1$ in both convergent-divergent channels. In Figure 4(a) at $\alpha=\pi / 4$ for divergent channel as $\phi$ increases, the center line velocity increases and the backflow is observed near the walls. 


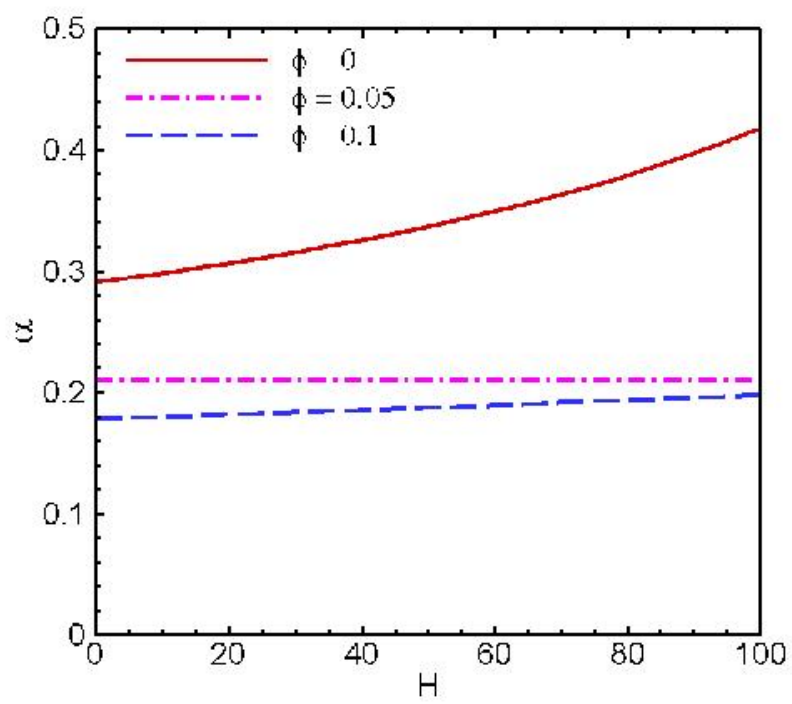

Fig. 9: Critical relation between $\alpha$ and $H$ for different values of $\phi$ at $\operatorname{Re}=20$ obtained by HPDA [28] for $\mathrm{d}=$ 4.

Conversely, Figure 4(b) for convergent channel $(\alpha=-\pi / 4)$ represents that backflow starts at the centerline when $(\phi=0.05)$ whether there is no sign of backflow in absence of nanoparticles $(\phi=0)$ and these properties are enhanced with rising $\phi=0.1$. Therefore, the state of backflow in convergent channel is completely opposite in comparison to divergent channel.

Figure 5 predicts the combined effects of magnetic field and nanoparticles volume fraction on the velocity for divergent channel with fixed Reynolds number. The figure represents sensible decreases in the centerline velocity with rising Hartmann number for both viscous and nanofluid that coincide with those results of [20]. It is also observed that for all values of Hartmann number there is no backflow in the viscous fluid $(\phi=0)$, nevertheless backflow starts for nanofluid with $H=0$ at $\alpha=0.1, \operatorname{Re}=7$ and this phenomenon is decreased with the rising values of Hartmann number. Employing the algebraic approximation method to the series (14) we have obtained the bifurcation graphs of $\alpha$ and $\operatorname{Re}$.

Figure 6 shows the bifurcation diagram of $\alpha$ with the effect of nanofluid. It is interesting to notice that there are two solution branches of velocity when $\alpha<\alpha_{c}$, one solution when $\alpha=\alpha_{c}$, and no solution when $\alpha>\alpha_{c}$, where $\alpha_{c}$ is the critical value of $\alpha$ for which the solution exists. It can be also noted here that the bifurcation points change from $\alpha \approx 0.2691819115$ to $\alpha \approx 0.2122678984$ and then to $\alpha \approx 0.1963360739$ for different values of nanoparticles volume fraction respectively at $\operatorname{Re}=20, H=1$. Moreover, from Figure 7 it is observed that the flow also bifurcates at $\operatorname{Re}=\operatorname{Re}_{c}$. On the other hand, the bifurcation points decreases uniformly for three different values of nanoparticles volume 
fraction at $\alpha=0.1, H=1$. The conjecture of Figures 6 and 7 is consistent with the results shown in Tables 1 and 2 using differential approximation. One major finding is that, as nanoparticles volume fraction increases the critical channel angle and flow Reynolds number decreases. The temporal and spatial complexity of observed flows changes in a succession of bifurcations until the onset of instability. Each bifurcation is marked by the onset of instability of one flow and followed by equilibrium to another stable flow. The High-order partial Differential Approximant HPDA [28] is applied to the series (14) in order to determine the critical relationship among the parameters $\alpha, H$ and Re with the effect of nanofluid. Figure 8 displays the critical relation between the channel angular width $\alpha$ and flow Reynolds number Re for three various values of $\mathrm{Cu}$-nanoparticles volume fraction.

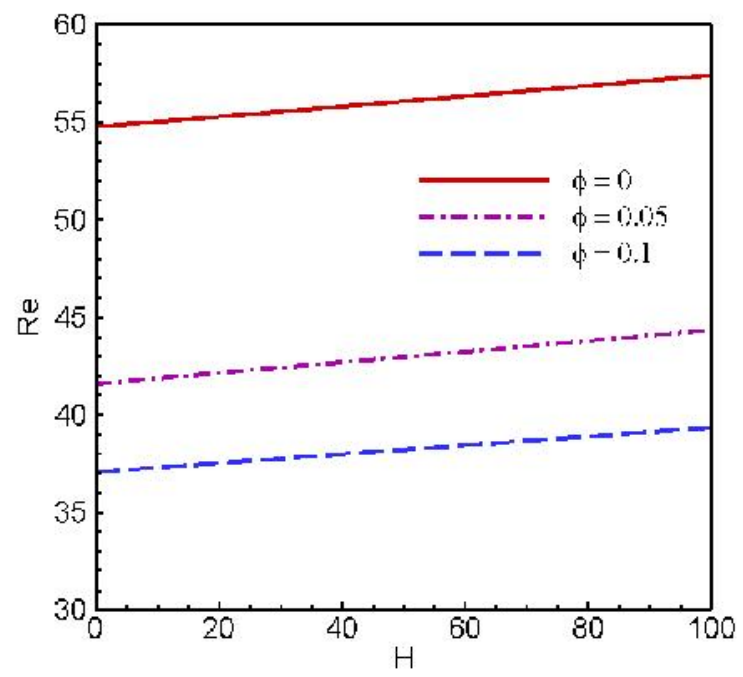

Fig. 10: Critical relation between Re and $H$ for different values of $\phi$ at $\alpha=0.1$ obtained by HPDA [28] for $\mathrm{d}=4$.

It is found that as $\alpha$ increases then Re decreases and conversely Re increases when $\alpha$ decreases. This implies that both channel angle and Reynolds number are inversely proportional to each other which are an excellent agreement with previously established results obtained by Fraenkel [3] for $\phi=0$ in classical Jeffery-Hamel flow. A significant variation is observed in the relationship curves of $\mathrm{Cu}$-nanoparticles for $\phi=0.05 \& \phi=0.1$. Figure 9 depicts the relationship between $\alpha$ and Hartmann number $H$ with different volume fraction of nanoparticles in divergent channel. It is seen from the figure that $\alpha$ increases as $H$ increases and the rate of increase is lower in $\mathrm{Cu}$-water nanofluid. However, $\alpha$ decreases as $\phi$ increases. Finally, in Figure10, the flow Reynolds number $R e$ increases with rising $H$ but then the tendency of increase becomes slower for $\phi=0.05 \& \phi=0.1$. The conjecture of both the Figures 9 and 10 coincides with those results of Alam and Khan [14] at $\phi=0$. Therefore, nanofluid diminishes the alternation phenomenon in the relationship graphs among the parameters. Series analysis plays an important role in many areas, particularly in fluid dynamics, where, as mentioned earlier, the presence of real 
singularities in the solution may reflect some changes in the nature of the flow. The criticality of channel angle and flow Reynolds number lead to instability in the fluid flow with a significant effect of nanofluid.

\section{Conclusion}

The magnetohydrodynamic Jeffry-Hamel flow problem with the $\mathrm{Cu}$-water nanofluid is investigated using a special type of Hermite-Padé approximation technique. A comparison is made between the available results and the present approximate solutions. The accurate numerical approximation of the critical parameters of the flow is obtained. The influence of various physical parameters on the velocity is discussed in detail. The basic conclusions are as follows:

$>$ Increasing channel semi angle leads to enrichment of fluid centerline velocity in the channel for both base fluid and nanofluid.

$>$ Increasing Hartmann number reduces fluid flow in the channel centerline and produces the backflow reduction near the walls for both base fluid and nanofluid.

$>$ The velocity increases as nanoparticles volume fraction increases along the centerline whereas increasing volume fraction generates backflow near the walls.

$>$ The dominating singularity behaviour is a branch point singularity with the critical exponent half for both the wall divergence semi-angle $\alpha$ and flow Reynolds number $R e$ is found with the effect of nanoparticles volume fraction.

$>$ The critical relationship among the parameters with the effect of nanofluid coincides with the conjecture of classical Jeffery-Hamel flow.

The computational cost of the series coefficients of the solution is very high in this problem. Hermite-Padé approximation technique could produce better and accurate results if more terms of the series were computed using highly powerful computer. However, we provide a basis for guidance about new approximants idea for summing power series that should be chosen for many problems in fluid mechanics and similar subjects.

\section{Acknowledgement}

This work is done within the framework of the $\mathrm{PhD}$ program of the first named author (corresponding author) under Department of Mathematics, Bangladesh University of Engineering and Technology, Dhaka. Financial support in the form of Bangabandhu Fellowship in Science and ICT project is gratefully acknowledged.

\section{REFERENCES}

[1] Jeffery, G.B. (1915), The Two-Dimensional Steady Motion of a Viscous Fluid, Philosophical Magazine, Vol. 6, pp. 455-465.

[2] Hamel, G. (1916), Spiralförmige Bewgungen Zäher Flüssigkeiten, Jahresbericht der Deutschen Math. Vereinigung, Vol. 25, pp. 34-60. 
[3] Fraenkel, L.E. (1962), Laminar flow in symmetrical channels with slightly curved walls. I: On the Jeffery-Hamel solutions for flow between plane walls, Proceeding of the Royal Society of London, Vol. 267, pp. 119-138.

[4] Sobey, I.J. and Drazin, P.G. (1986), Bifurcations of two-dimensional channel flows, Journal of Fluid Mechanics, Vol. 171, pp. 263-287.

[5] Makinde, O.D. (1997), Steady flow in a linearly diverging asymmetrical channel, Computer Assisted Mechanics and Engineering Sciences, Vol. 4, pp. 157 - 165.

[6] Cha, J. E., Ahn, Y. C. and Kim, M. H. (2002), Flow measurement with an electromagnetic flow meter in two-phase bubbly and slug flow regimes, Flow Measurement and Instrumentation. Vol. 12, No. 56, pp. 329-339.

[7] Tendler, M. (1983), Confinement and related transport in extrap geometry, Nuclear Instruments and Methods in Physics Research, Vol. 207, No. 1-2, pp. 233-240.

[8] Makinde, O.D. and Mhone, P.Y. (2006), Hermite-Pade' Approximation approach to Hydromagnetic flows in convergent-divergent channels, Applied Mathematics and Computations, Vol. 181, No. 2, pp. 966-972.

[9] Esmaili, Q., Ramiar, A., Alizadeh, E. and Ganji, D.D. (2008), An approximation of the analytical Solution of the Jeffery-Hamel flow by decomposition method, Physics Letters A, Vol. 372, pp. 34343439.

[10] Joneidi, A.A., Domairry, G. and Babaelahi, M. (2010), Three analytical methods applied to JefferyHamel flow, Commun. Nonlinear Sci. Numer. Simulat., Vol. 15, pp. 3423-3434.

[11] Axford, W.I. (1961), The Magnetohydrodynamic Jeffrey-Hamel problem for a weakly conducting fluid, Q. J. Mech. Appl. Math., Vol. 14, pp. 335-351.

[12] Motsa, S.S., Sibanda, P., Awad, F.G. and Shateyi, S. (2010), A new spectral-homotopy analysis method for the MHD Jeffery-Hamel problem, Computers \& Fluids, Vol. 39, pp. 1219-1225.

[13] Moghimia, S.M., Ganji, D.D., Bararnia, H., Hosseini, M. and Jalaal, M. (2011), Homotopy perturbation method for nonlinear MHD Jeffery-Hamel Problem, Computers and Mathematics with Applications, Vol. 61, pp. 2213-2216.

[14] Alam, M.S. and Khan, M.A.H. (2010), Critical behaviour of the MHD flow in convergent- divergent channels, Journal of Naval Architecture and Marine Engineering, Vol. 7, No. 2, pp. 83-93.

[15] Choi, S.U.S. (1995), Enhancing thermal conductivity of fluids with nanoparticles, Proceedings of the ASME International Mechanical Engineering Congress and Exposition, FED 231/MD. Vol. 66, ASME, San Francisco, USA, pp. 99-105.

[16] Oztop, H.F. and Abu-Nada, E. (2008), Numerical study of natural convection in partially heated rectangular enclosures filled with nanofluids, Int. J. Heat Fluid Flow, Vol. 29, pp. 1326-1336.

[17] Das, S.K., Choi, S.U.S., Yu, W. and Pradeep, T. (2007), Nanofluids: Science and Technology, Wiley, New York.

[18] Das, S.K., Choi, S.U.S. and Patel, H.E. (2006), Heat transfer in nanofluids-a Review, Heat Transfer Eng., Vol. 27, No. 10, pp. 3-19.

[19] Rahman, M.M., Rosca, A.V. and Pop, I. (2014), Boundary layer flow of a nanofluid past a permeable exponentially shrinking surface with second order slip using Buongiorno's model, International Journal of Heat and Mass Transfer, Vol. 77, pp. 1133-1143.

[20] Sheikholeslami, M., Ganji, D.D., Ashorynejad, H.R. and Rokni, H.B. (2012), Analytical investigation of Jeffery-Hamel flow with high magnetic field and nanoparticle by Adomian decomposition method, Appl. Math. Mech. Eng., Vol. 33, pp. 25-36.

[21] Moradi, A., Alsaedi, A. and Hayat, T. (2013), Investigation of Nanoparticles Effect on the JefferyHamel Flow, Arab J. Sci. Eng., Vol. 38, pp. 2845-2853.

[22] Khan, M.A.H. (2001), Singularity Analysis by Summing Power Series, PhD Thesis. University of Bristol, England. 
[23] Aminossadati, S.M. and Ghasemi, B. (2009), Natural convection cooling of a localized heat source at the bottom of a nanofluid-filled enclosure, Eur. J. Mech. B/Fluids, Vol. 28, pp. 630-640.

[24] Padé, H. (1892), Sur la représentation approchée d'une fonction pour des fractions rationnelles, Ann. Sci. École Norm. Sup. Suppl., Vol. 9, pp. 1-93.

[25] Hermite, C. (1893), Sur la généralisation des fractions continues algébriques, Annali di Mathematica Pura e Applicata, Vol. 21, No. 2, pp. 289-308.

[26] Drazin, P.G. and Tourigny, Y. (1996), Numerically study of bifurcation by analytic continuation of a function defined by a power series, SIAM Journal of Applied Mathematics, Vol. 56, pp. 1-18.

[27] Khan, M.A.H. (2002), High-Order Differential Approximants, Journal of Computational and Applied Mathematics, Vol. 149, pp. 457-468.

[28] Rahman, M.M. (2004), A New Approach to Partial Differential Approximants, M. Phil thesis, Bangladesh University of Engineering \& Technology, Dhaka. 